EPJ Web of Conferences 88, 00028 (2015)

DOI: $10.1051 /$ epjconf/ 20158800028

(C) Owned by the authors, published by EDP Sciences - SIF, 2015

\title{
Isospin effects in fragmentation reactions
}

\author{
J. Benlliure
}

Universidade de Santiago de Compostela, 15786 Santiago de Compostela, Spain

\begin{abstract}
Fragmentation reactions induced by relativistic heavy ions are characterized by large fluctuations in excitation energy and isospin. These features justifies the interest of this reaction mechanism for the production of nuclei far from stability. A particularly interesting reaction channel proving those fluctuations is the nucleon removal. Moreover, those processes are of interest to characterize the structural properties of the nuclei involved in the reactions.
\end{abstract}

\section{Introduction}

Peripheral or mid-peripheral heavy-ion collisions at kinetic energies above the Fermi energy and up to few GeVs per nucleon lead to the fragmentation of the projectile and target nuclei. Such conditions justify the use of the participant-spectator picture [1] and the two-stage reaction scheme [2] for the description of this reaction mechanism. According to these models the fragmentation of projectile and target nuclei can be understood as a two-step process. In the first step, spectators lose nucleons and gain excitation energy and some angular momentum according to the impact parameter of the collision. In the second stage, these pre-fragments evacuate the excitation energy and angular momentum by emitting nucleons, clusters of nucleons or gamma rays. In heavy fragments fission may also compete with the other de-excitation channels.

The general characteristics of fragmentation reactions are relatively well known since the 80s [3]. However, the large dynamical and isospin range covered by this reaction mechanism together with the use of advanced detection 
setups are key features that justify the present importance of fragmentation reactions in nuclear physics research [4]. This reaction mechanism is, for example, well suited for the investigation of nuclear matter at extreme conditions. The investigation of the multifragmentation process [5] and the characterization of the symmetry energy at low densities [6,7], but covering a large range in isospin are some key examples.

Fragmentation reactions are also suitable for the in-flight production of nuclei far from stability. The fluctuations in mass, neutron excess and excitation energy in the pre-fragments are exploited to reach very exotic nuclear species.

Finally fragmentation also has an important impact in some applications. The most clear example is radiotherapy using relativistic heavy ions.

The present document is organised as follows. First we will briefly discuss the main features characterizing the production of final residues in fragmentation reactions. We will then discuss the impact of fragmentation in the production of nuclei far from stability. Finally we will present recent results concerning the investigation of the most peripheral fragmentation reactions, nucleon removal, with projectiles covering a large range in isospin.

\section{Key features of fragmentation reactions}

One of the main features of fragmentation reactions is that the nature and yields of the final fragmentation residues is very much correlated with the impact parameter and almost independent on the initial kinetic energy of the projectile nuclei. This independence on the initial kinetic energy is know as the "limiting fragmentation" regime [8]. Therefore the large range in mass and excitation energy of the spectators produced in relativistic heavy ion collisions is directly determined by the impact parameter. Indeed, midperipheral collisions may lead to pre-fragments losing up to $1 / 3$ the the initial mass of the colliding nuclei and with temperatures as high as $10 \mathrm{MeV}$.

The neutron excess in the pre-fragments is determined by the fact that the abrasion of nucleons during the first stage of the collision is an isospin independent process. Therefore the final relative content in protons and neutrons of the pre-fragments can be describe by an hypergeometrical distribution [9]. The main characteristics of this distribution are the relatively large fluctuations as shown in Fig. 1. In the left panel of this figure we display on top of a chart of nuclides the calculated isotopic composition of the projectile pre-fragments produced in collisions induced by ${ }^{197} \mathrm{Au}$ projectiles impinging on beryllium target at $1 \mathrm{~A} \mathrm{GeV}$. As can be observed those 

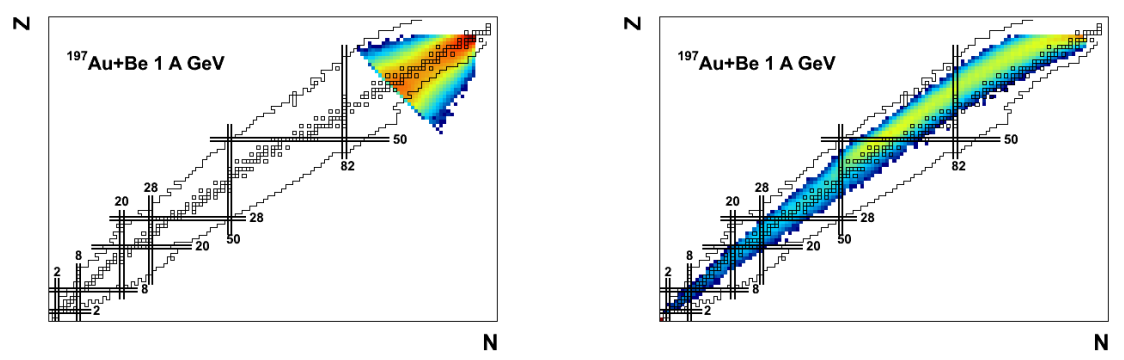

Figure 1: Left panel: Calculated isotopic composition of the projectile prefragments produced during the abrasion stage in the fragmentation reaction ${ }^{197} \mathrm{Au}$ on $\mathrm{Be}$ at $1 \mathrm{~A} \mathrm{GeV}$ on top of a chart of nuclides. Right panel: Calculated isotopic composition after de-excitation process.

pre-fragments cover a range in isospin broaden that the present limits of the chart of nuclides. Therefore, the abrasion process is governed by extremely large fluctuations in neutron excess. The fluctuations in the final fragments are however, very much reduced by the subsequent de-excitation process as shown in the left panel of Fig. 1.

The de-excitation of the hot remnants produced during the abrasion stage is mostly described by nucleon evaporation. Because this process is governed by the respective binding energies of protons and neutrons, together with the Coulomb barrier of protons, the isospin equilibrium is reached at the left of the valley of stability where neutron binding energies equal proton binding energies plus the corresponding Coulomb barrier, defining the so called "evaporation corridor". This process also depopulates the pre-fragments with the lowest proton and neutron binding energies and consequently the largest isospin asymmetries.

In summary, we can conclude that fragmentation reactions produce nuclei covering a large range in excitation energy, mass and neutron excess. However, the de-excitation process limits the isospin range according to binding energies.

\section{Production of nuclei far from stability}

The large dispersion in mass and neutron excess of the residual nuclei produced in fragmentation reactions makes this mechanism suitable for the production of nuclei far from stability. The most populated region of the chart of nuclides using fragmentation reactions is the one located at the 
left of the line of $\beta$ stability corresponding to the "evaporation corridor". Therefore, fragmentation produces mostly neutron-deficient fragments, as shown in the right panel of Fig. 1. However, the survival of the large fluctuations in neutron excess and excitation energy of the pre-fragments produced during the abrasion phase broadens the isotopic distributions of the fragments. A particularly interesting reaction channel is the so called "cold fragmentation" [10]. These are processes where the colliding nuclei only lose protons or neutrons, during the abrasion phase, and the excitation energy gained is below the particle evaporation threshold. These reactions produce then the most neutron- or proton-rich nuclei accessible with a given projectile nucleus. In this way the proton drip-line can be easily reached and very neutron-rich nuclei can be produced. In both cases, the final production yields will decrease strongly with the binding energies of protons and neutrons, respectively.

Fragmentation reactions allows to reach the proton drip-line up to around mercury. For heavier elements fission depopulates the production of neutron-deficient isotopes [11]. Unfortunately this limitation is common to any other reaction mechanism one could use to produce heavy neutrondeficient nuclei as for example fusion-evaporation reactions.

This reaction mechanism is also commonly used to produce neutron-rich nuclei, in particular light ones. In this region of the chart of nuclides important progress was achieved by fragmenting neutron-rich ${ }^{48} \mathrm{Ca}$ projectiles at NSCL [12]. Indeed, this reaction allowed to produce the heaviest known nuclei at the neutron drip-line, ${ }^{40} \mathrm{Mg}$ and ${ }^{42} \mathrm{Al}$ [13].

Medium-mass neutron-rich nuclei can also be produced by fragmentation of stable beams such as ${ }^{136} \mathrm{Xe}$ [14]. A two-step reaction scheme for the production of medium-mass neutron-rich nuclei was proposed some years ago [15]. According to this proposal neutron-rich fission fragments could be reaccelerated and fragmented. Following this idea, the fragmentation of ${ }^{132} \mathrm{Sn}$ projectiles has been investigated at GSI [16]. However, fission reactions are considered since long ago as the most competitive reaction mechanism to produce nuclei in this region of the chart of nuclides [17]. Indeed, the most neutron-rich nuclei in this region of the chart of nuclides were recently obtained at RIKEN making use of the high beam intensities delivered by the new facility [18].

The region corresponding to heavy neutron-rich nuclei is rather challenging. Actually, the limits of the chart of nuclides in this region were rather close to stability until recently. Heavy neutron-rich nuclei can only be produced by multi-nucleon transfer or fragmentation reactions, although the latter allows to produce a larger variety of final species. However, the major 


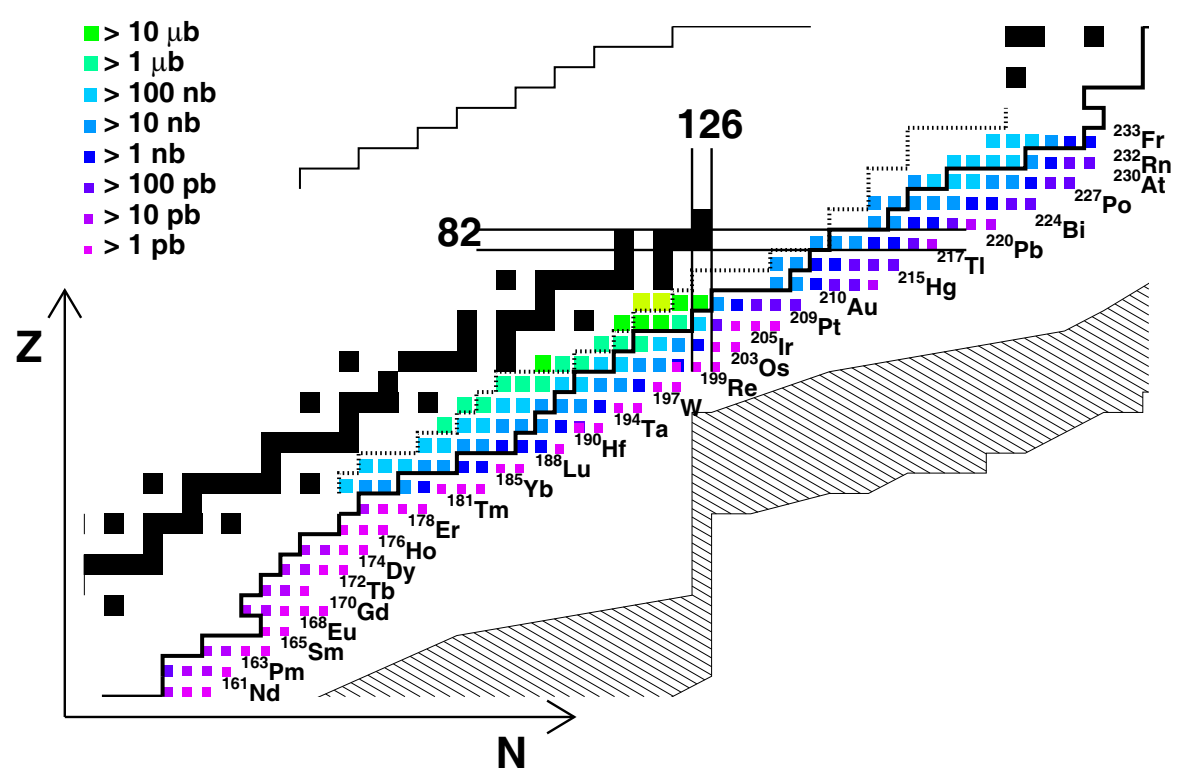

Figure 2: Chart of nuclides indicating the heavy neutron-rich nuclei that have been produced at GSI during the last few years in reactions induced by ${ }^{238} \mathrm{U}$ and ${ }^{208} \mathrm{~Pb}$ projectiles impinging onto a beryllium target at $1000 \mathrm{~A} \mathrm{MeV}$.

challenge in this region is the unambiguous identification of the final nuclei. In fact, the large atomic number increases the possibility that the produced nuclei are not fully stripped when leaving the target material. Those nuclei have an $\mathrm{A} / \mathrm{Q}$ value similar to the one of more neutron-rich isotopes fully stripped. In particular, (A - 3, Z) residues with a hydrogen-like ionic charge state $(\mathrm{Q}=\mathrm{Z}-1)$ have a similar magnetic rigidity as fully stripped $(\mathrm{A}, \mathrm{Z})$ nuclei. Since the neutron-rich (A - 3, Z) fragments typically have production cross sections around two orders of magnitude larger than the cross sections of the isotopes with three more neutrons (A, Z), a few percent of the production of $(\mathrm{A}-3, \mathrm{Z})$ fragments with a hydrogen-like ionic charge state could represent almost $100 \%$ contamination in (A, Z) fully stripped nuclei.

This limitation justifies why the most important progress in extending the limits of the chart of nuclides in this region has been obtained at GSI. The relativistic energies reduce the probability of charge states while the combined used of a high-resolving power magnetic spectrometer such as the Fragment Separator and stripping foils make possible the unambiguous identification of fully stripped residual nuclei [19]. Using this technique, and beams of ${ }^{238} \mathrm{U}$ and ${ }^{208} \mathrm{~Pb}$, it was possible to produce during the last 
years around 126 new neutron-rich nuclei [20-22]. As indicated in Fig. 2, these experiments have allowed a clear extension of the chart of nuclei in that particular region, reaching almost the r-process path. Moreover, the production cross sections for all measured nuclei and the half lives for some of them were also determined in these experiments. The cross sections have been used to benchmark model calculations predicting the fragmentation yields that one can expect in future RIB facilities. Half lives indicate the clear role of first forbidden transitions in heavy neutron-rich nuclei across $\mathrm{N}=126[23,24]$.

\section{Isospin effects in nucleon-removal processes}

A particularly interesting process is the nucleon removal that can be considered as the most peripheral fragmentation channel. Isospin effects in these reactions are extremely important for the understanding of the production of the most exotic nuclear species, as discussed in the previous sections, but also because these reactions are used as spectroscopic tools providing structural information on the participant nuclei. With respect to this latter topic, the understanding of the quenching of the spectroscopic factors obtained in the removal of deeply bound nucleons [25] is still an open question.

To investigate isosopin effects in nucleon removal processes, a dedicated experiment was recently performed at GSI. In this experiment the nucleon removal from stable $\left({ }^{112} \mathrm{Sn}\right.$ and $\left.{ }^{124} \mathrm{Sn}\right)$ and unstable $\left({ }^{110} \mathrm{Sn},{ }^{120} \mathrm{Sn}\right.$ and $\left.{ }^{124} \mathrm{Sn}\right)$ tin isotopes at $1000 \mathrm{~A} \mathrm{MeV}$ was investigated [26]. The knockout of stable nuclei was induced by a carbon target placed at the entrance of the fragment separator. Unstable projectiles were produced by fragmenting the stable ones at the entrance of the Fragment Separator in a beryllium target. The isotopes of interest were then identified with the first section of the Fragment Separator and the knockout was induced by another carbon target located at the intermediate image plane of the spectrometer and the reaction residues analysed in the second half.

The cross sections for the proton removal channels measured in this experiment are reported in Fig. 3 together with data obtained at RIKEN [27] for ${ }^{104} \mathrm{Sn}$ and ${ }^{112} \mathrm{Sn}$ at 155 and $173 \mathrm{~A} \mathrm{MeV}$, respectively. As can be seen in the figure, the measurements for ${ }^{112} \mathrm{Sn}$ performed at GSI at $1000 \mathrm{~A} \mathrm{MeV}$ and at RIKEN at $173 \mathrm{~A} \mathrm{MeV}$ are in very good agreement, confirming that in both cases the reaction reaches the "limiting fragmentation" regime. We also observe that for most of the tin isotopes we could determine the one and two proton-removal cross sections and in the case of ${ }^{112} \mathrm{Sn}$ we measured 


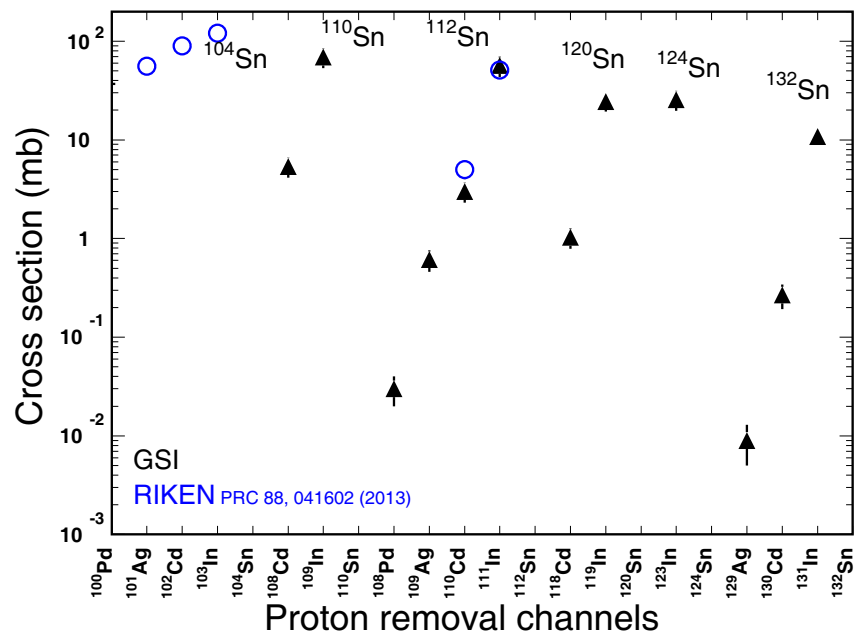

Figure 3: Cross sections of the proton-removal processes measured at GSI for different tin isotopes on carbon at $1000 \mathrm{~A} \mathrm{MeV}$ together with data obtained at RIKEN [27] for ${ }^{104} \mathrm{Sn}$ and ${ }^{112} \mathrm{Sn}$ at 155 and $173 \mathrm{~A} \mathrm{MeV}$, respectively.

the removal of up to four protons. The analysis of the evolution of the cross sections with the isospin of the projectile clearly indicates that the cross section of the proton knockout decreases with the neutron excess. This is clearly seen for the one proton knock-out but also in the slope defining the evolution of the cross sections for the multiple knockout processes.

In Fig. 4 we report the cross sections of the one proton knockout (left panel) and one neutron knockout (right panel) from different tin isotopes as a function of their neutron excess. In this figure the solid triangles represent data from GSI and the open circles data from RIKEN. The figure confirms that the cross sections of the knockout of deficient nucleons, protons in neutron-rich systems and neutrons in neutron-deficient ones, decrease with the neutron-proton asymmetries. This behaviour coincides with the evolution of the spectroscopic factors discussed above.

A possible reason explaining the evolution of these cross sections is an increase in the excitation energy induced in the remnants by the knockout of deeply bound nucleons. This possibility was already proposed by Louchart and collaborators [28].

To test this ideas we have performed calculations with the abrasionablation model ABRABLA [29]. In this model the mass of the reaction pre-fragments is obtained from the geometrical overlap between projectile 

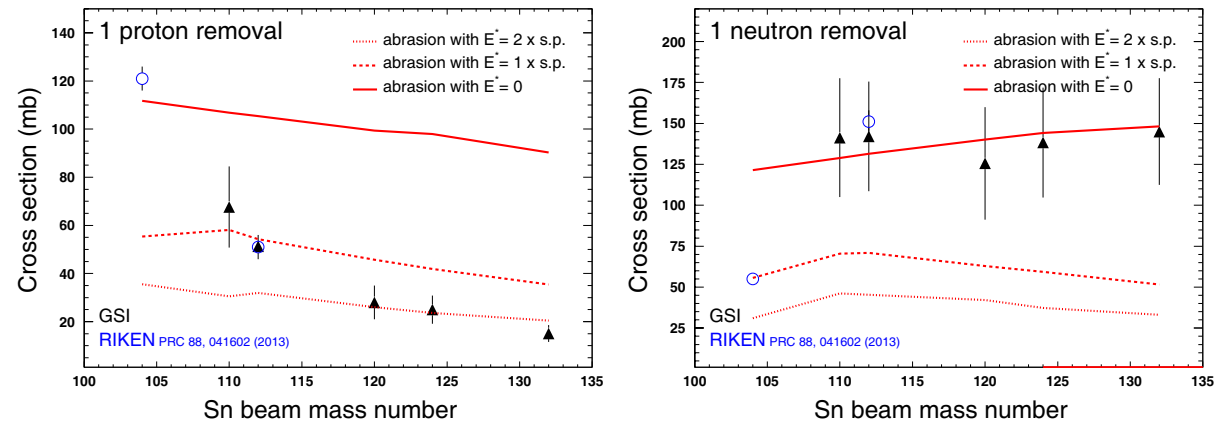

Figure 4: Evolution of the cross sections of the one proton knockout (left panel) and one neutron knockout (right panel) from different tin isotopes as a function of their neutron excess. The lines represent predictions obtained with the code ABRABLA, see text for details.

and target, their neutron-to-proton ratio from an hypergeometrical distribution of the probability to remove a proton or a neutron from an ensemble of $\mathrm{N}$ neutrons and $\mathrm{Z}$ protons and the excitation energy form particle-hole excitations in the Fermi distribution of nucleons inside the nucleus.

In Fig. 4 we display the results of three predictions obtained with this code. In the first one (dotted line) we neglect the excitation energy gained by the pre-fragments in the knockout process, in the second one (dashed line) we consider the excitation energy according to the particle-hole picture for a potential depth of $40 \mathrm{MeV}$ and in the third one (solid line) we increase the excitation energy gained by the remnants by a factor two, according to the results presented in [30]. As can be seen, the loosely bound nucleons can be described almost with no excitation energy while for the deeply bound ones we even need to increase the excitation energy predicted by the particle-hole excitations by a factor two. This result would support the importance of the remnants excitation to describe correctly the cross sections of the knockout processes.

The previous conclusions are based on rather simplistic model calculations. Detailed calculations with a realistic description of the nuclear potential and the level structure of the corresponding nuclei according to isospin are then required. 


\section{Conclusions}

The renewed interest in fragmentation reactions is justified by the use of this reactions for the production of nuclei far from stability based on the in-flight production technique, the possibility to extend previous studies concerning the EOS and in particular, the symmetry energy using asymmetric nuclear matter, and finally because of the use of these reactions is some key applications as heavy-ion radiotherapy. All these areas of interest benefit form the specific features characterising the production of the final fragments which are the large range in mass and the large fluctuations in isospin and excitation energy covered by this reaction mechanism.

During the last years tremendous progress has been made in producing extremely exotic nuclei using fragmentation reactions, in particular in the neutron-rich sector. In this area the drip line has been reached until aluminium, and many new heavy neutron-rich nuclei has been synthesized.

The investigation of isospin effects in nucleon removal channels are particularly interesting to characterize the fluctuations governing fragmentation reactions, but also to contribute to the understanding of the puzzling quenching of the spectroscopic factors obtained in the removal of deeply bound nucleons. A dedicated experiment recently performed at GSI point at the role of the excitation energy gained by the remnants produced in these reactions channels, although state-of-the-art model calculations should confirm these conclusions.

\section{References}

[1] R.J. Glauber, Lectures in Theoretical Physics, Vol. 1, ed. W.E. Brittin and L.G. Dunham, (Interscience Publishers, NY, 1959) pp. 315-414.

[2] R. Serber, Phys. Rev. 72, 1114 (1947)

[3] J. Hüfner, Phys. Rep. 125, (1985)

[4] J. Benlliure, Proc. of the 10th International Nuclear Physics. Vancouver, Canada, July 4-9 2010. J. of Phys. Conf. 312, 082001 (2011)

[5] W. Trautmann et al., Nucl. Phys. A 787, 575 (2007)

[6] A. Le Fevre et al., Phys. Rev. Lett. 94, 162701 (2005)

[7] S. Kumar and Y.G. Mag, Phys. Rev. C 86, 051601 (2012) 
[8] E.M. Friedlander and H.H. Heckmann, in Treatise on Heavy Ion Science, edited by D.A. Bromley (Plenum, New York, (1985), Vol. 4, p.403

[9] L.F. Oliveira, R. Donangelo and J.O. Rasmussen, Phys. Rev. C 19, 826 (1979)

[10] J. Benlliure et al., Nucl. Phys. A 660, 87 (1999)

[11] J. Kurcewicz et al., Nucl. Phys. A 767,1 (2006)

[12] M. Mocko et al., Phys. Rev. C 74, 05412 (2006)

[13] T. Baumann et al., Nature 449, 1022 (2007)

[14] J. Benlliure et al., Phys. Rev. C 78, 054605 (2008)

[15] K. Helariutta et al., Eur. Phys. J. A 17, 181 (2003)

[16] D. Perez-Loureiro et al., Phys. Lett. B 703, 552 (2011)

[17] M. Bernas et al., Phys. Lett. B 331, 19 (1994)

[18] T. Ohnishi et al., J. Phys. Sc. Jpn. 79, 073201 (2010)

[19] E. Casarejos et al., Phys. Rev. C 74, 044612 (2006)

[20] H. Alvarez-Pol et al., Phys. Rev. C 82, 041602(R) (2010)

[21] J. Kurcewicz et al., Phys. Lett. B 717, 371 (2012)

[22] T. Kurtukian-Nieto et al., Phys. Rev. C 89, 024616 (2014)

[23] A.I. Morales et al., Phys. Rev. Lett. 113, 022702 (2014)

[24] T. Kurtukian-Nieto et al., Eur. Phys. J. A 50, 135 (2014)

[25] A. Gade et al., Phys. Rev. C 77, 044306 (2008)

[26] J. Vargas, PhD thesis, University of Santiago de Compostela, Spain (2014)

[27] L. Audirac et al., Phys. Rev. C 88, 041602(R) (2013)

[28] C. Louchart et al., Phys. Rev. C 83, 011601(R) (2011)

[29] J.-J. Gaimard, K.-H. Schmidt, Nucl. Phys. A 531, 709 (1991)

[30] K.-H. Schmidt et al., Phys. Lett. B 300, 313 (1993) 